\title{
CRITICAL PARTITIONS AND NODAL DEFICIENCY OF BILLIARD EIGENFUNCTIONS
}

\author{
GREGORY BERKOLAIKO ${ }^{1}$, PETER KUCHMENT ${ }^{1}$, AND UZY SMILANSKY ${ }^{2,3}$
}

\begin{abstract}
The paper addresses the nodal count (i.e., the number of nodal domains) for eigenfunctions of Schrödinger operators with Dirichlet boundary conditions in bounded domains. The classical Sturm theorem states that in dimension one, the nodal and eigenfunction counts coincide: the $n$ th eigenfunction partitions the interval into $n$ nodal domains. The Courant Nodal Theorem claims that in any dimension, the number of nodal domains $\nu_{n}$ of the $n$th eigenfunction cannot exceed $n$. However, it follows from an asymptotically stronger upper bound by Pleijel that in dimensions higher than 1 the equality can hold for only finitely many eigenfunctions. Thus, in most cases a "nodal deficiency" $d_{n}=n-\nu_{n}$ arises. One can say that the nature of the nodal deficiency has not been understood.

It was suggested in recent years that, rather than starting with eigenfunctions, one can look at partitions of the domain into $\nu$ sub-domains, asking which partitions can correspond to eigenfunctions, and what would be the corresponding deficiency. To this end one defines an "energy" of a partition, for example, the maximum of the ground state energies of the sub-domains. One notices that if a partition does correspond to an eigenfunction, then the ground state energies of all the nodal domains are the same, i.e., it is an equipartition. It was shown in a recent paper by Helffer, Hoffmann-Ostenhof and Terracini that (under some natural conditions) partitions minimizing the energy functional correspond to the "Courant sharp" eigenfunctions, i.e. to those with zero nodal deficiency.

In this paper it is shown that it is beneficial to restrict the domain of the functional to the equipartition, where it becomes smooth. Then, under some genericity conditions, the nodal partitions correspond exactly to the critical points of the functional. Moreover, the nodal deficiency turns out to be equal to the Morse index at the corresponding critical point. This explains, in particular, why the minimal partitions must be Courant sharp.
\end{abstract}

\section{INTRODUCTION}

We consider the Schrödinger operator

$$
H=-\Delta+V(x)
$$

with Dirichlet conditions in a connected bounded domain $\Omega \subset \mathbb{R}^{d}$. We will assume that the domain has a smooth boundary and that the real potential $V$ is also smooth on $\bar{\Omega}$. While these assumptions are overly restrictive, we do not want to burden our considerations with less significant details. See the final section for additional remarks.

The operator $H$ can be defined via its quadratic form

$$
h[u, u]=\int_{\Omega}|\nabla u(x)|^{2} d x+\int_{\Omega} V(x)|u(x)|^{2} d x
$$


with the domain $H_{0}^{1}(\Omega)$. Thus defined, it is self-adjoint in $L_{2}(\Omega)$ and has real discrete spectrum of finite multiplicity

$$
\lambda_{1}<\lambda_{2} \leq \lambda_{3} \leq \ldots,
$$

where $\lim _{n \rightarrow \infty} \lambda_{n}=\infty$. It has an orthonormal basis of real-valued eigenfunctions $\psi_{n}$ such that $\psi_{1}(x)>0$. We will sometimes use the notations $H(\Omega)$ and $\lambda_{j}(\Omega)$, when we need to emphasize the dependence of the operator and its spectrum on the domain.

Definition 1. For a function $f(x)$ we will be interested in its nodal (zero) set

$$
\mathcal{Z}(f):=f^{-1}(0)=\overline{\{x \in \Omega \mid f(x)=0\}} .
$$

The complement $\Omega \backslash N(f)$ is the union of connected open sub-domains $D_{1}, \ldots, D_{\nu}$ of $\Omega$, which we will call nodal domains. The nodal domains form the nodal partition $P(f)=\left\{D_{j}\right\}$ corresponding to the function.

We will be mostly interested in the case when $f(x)$ is an eigenfunction $\psi_{n}$, and thus in its nodal set $\mathcal{Z}\left(\psi_{n}\right)$, its nodal domains $D_{1}, \ldots, D_{\nu}$ of $\Omega$, and its nodal partition $P\left(\psi_{n}\right)=\left\{D_{j}\right\}$.

A lot of attention has been paid to the nodal structure of eigenfunctions (e.g., [15, 19, 30, 43, 46] and references therein), and in particular to the number $\nu$ (or $\nu_{\psi_{n}}$, if one wants to emphasize dependence on the eigenfunction) of nodal domains of the $n$-th eigenfunction $\psi_{n}$ of $H$.

In spite of more than 300 years history of this topid1, open questions still abound. We will discuss here one of them, the issue of the so called nodal deficiency. The classical Sturm theorem states that in dimension one, the nodal and eigenfunction counts coincide: $\nu_{\psi_{n}}=n$. The Courant Nodal Theorem [14, Vol. I, Sec. V.5, VI.6] asserts that in any dimension, the upper bound on the number of nodal domains still holds:

$$
\nu_{\psi_{n}} \leq n
$$

While $\nu_{\psi_{1}}=1$ and $\nu_{\psi_{2}}=2$, it follows from an asymptotically stronger upper bound for $\nu_{\psi_{n}}$ by Pleijel [49] that in higher dimensions the equality $\nu_{\psi_{n}}=n$ can hold only for a finitely many values of $n$. Moreover, there are known example of eigenfunctions $\psi_{n}$ with arbitrarily large index $n$ that have just two nodal domains.

The eigenfunctions $\psi_{n}$ for which $\nu_{\psi_{n}}=n$, are sometimes called Courant sharp. The non-negative difference

$$
d_{n}:=n-\nu_{\psi_{n}}
$$

is said to be the nodal deficiency of an eigenfunction. It is believed that the integer sequence $\left\{d_{n}\right\}$ contains much information about the geometry of the domain (see, for example, [8, 10, 27, 37, 38]). However, we are not aware of any

\footnotetext{
${ }^{1}$ Robert Hooke observed on 8 July 1680 the nodal patterns on vibrating glass plates, running a bow along the edge of a glass plate covered with flour [47]. A hundred years later, the same effect was systematically studied by E. Chladni [11. In fact, such patterns were known to Leonardo da Vinci [42] and Galileo Galilei [23]. See also some historical discussion in [43].
} 
interpretation of the meaning of individual nodal deficiencies $d_{n}$. It is the goal of this text to present one such interpretation.

An important new approach has been developed in the last several years in the series of papers [13, 31, 32]. Namely, instead of concentrating on an eigenfunction, one can look at a partition of $\Omega$ by connected open domains $\left\{D_{j}\right\}_{j=1}^{\nu}$ and try to determine whether a given partition can be the nodal partition of an eigenfunction of a given operator $H$, and if yes, what could be the corresponding nodal deficiency.

Some necessary conditions on the partition are not hard to find. Indeed, one can introduce the graph of the partition, with each partition domain $D_{j}$ serving as a vertex and any two nodal domains that have a $(d-1)$-dimensional common boundary being connected by an edge. Since, due to standard uniqueness theorems for the Cauchy problem, the eigenfunction must change its sign when crossing any $(d-1)$-dimensional piece of the boundary of two adjacent nodal domains, we conclude that the following well known property holds:

Proposition 2. The graph of the nodal partition corresponding to an eigenfunction is bipartite.

Another important simple observation is:

Proposition 3. If $\left\{D_{j}\right\}_{j=1}^{\nu}$ is the nodal partition corresponding to the eigenfunction $\psi_{n}$ with eigenvalue $\lambda_{n}$, then for each nodal domain $D_{j}$, one has

$$
\lambda_{1}\left(D_{j}\right)=\lambda_{n}(\Omega) \text {. }
$$

(Here, as before, $\lambda_{k}(D)$ denotes the kth Dirichlet eigenvalue in the domain D.)

Indeed, by definition, $\psi_{n}$ does not change sign in $D_{j}$ and thus is proportional to the groundstate for $H\left(D_{j}\right)$.

This observation leads to the following notion that plays a crucial role in what follows:

Definition 4. A partition $P=\left\{D_{j}\right\}_{j=1}^{\nu}$ is said to be an equipartition, if the lowest eigenvalues of all operators $H\left(D_{j}\right)$ are the same, i.e.

$$
\lambda_{1}\left(D_{1}\right)=\lambda_{1}\left(D_{2}\right)=\cdots=\lambda_{1}\left(D_{\nu}\right) .
$$

As we have already mentioned, every nodal partition (i.e., the partition corresponding to an eigenfunction) is an equipartition.

This observation has lead to the following construction: given a natural number $\nu$, consider the "space" of "arbitrary" (with some natural restrictions) $\nu$-partitions $\left\{D_{j}\right\}$, i.e. partitions with $\nu$ sub-domains. Let us also introduce the functional

$$
\Lambda\left(\left\{D_{j}\right\}\right):=\max _{j=1, \ldots, \nu} \lambda_{1}\left(D_{j}\right)
$$

on this space.

One can look now at the minimal partitions, i.e. the partitions that minimize the functional $\Lambda\left(\left\{D_{j}\right\}\right)$ for a given $\nu$. Such minimal partitions are known to exist [13] and the following important result holds:

Theorem 5 (Helffer, Hoffmann-Ostenhof, Terracini [31]). Minimal bipartite partitions are exactly the nodal partitions of Courant sharp eigenfunctions. 


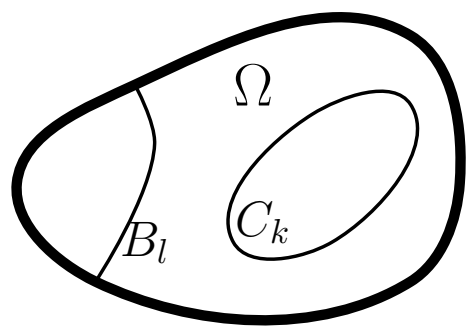

Figure 1. A generic partition

Our aim is to understand whether there is something that distinguishes the nodal partitions of the eigenfunctions that are not Courant sharp (which are the overwhelming majority) and what determines their nodal deficiencies. It was shown in a recent work [5] that in the quantum graph situation this question can be answered (see also [6] for the discussion of the discrete graph case). Inspired by this development, we address here the eigenfunctions of the operator $H$ defined above. As we will see, one has to consider not only the minima, but all the critical points of the functional $\Lambda$ on the "manifold" of equipartitions. Furthermore, it turns out that the Morse index of a critical point coincides with the nodal deficiency of the corresponding eigenfunction.

Before we formulate our assumptions we mention that generically with respect to perturbations of the potential $V(x)$ and/or the domain, the following conditions are satisfied (see [2, 3, 36, 44, 46, 51] and references therein).

Definition 6. We say the eigenfunction $\psi_{n}$ of $H$ is generic if

(1) The corresponding eigenvalue $\lambda_{n}$ is simple

(2) Zero is a regular value of the eigenfunction $\psi_{n}$ inside $\Omega$ (i.e., $\nabla \psi_{n}(x) \neq 0$ whenever $\left.\psi_{n}(x)=0\right)$. The normal derivative $\partial \psi_{n} / \partial n$ of the eigenfunction $\psi_{n}$ on the boundary of $\Omega$ has zero as its regular value (i.e., the tangential to $\partial \Omega$ gradient of $\partial \psi_{n} / \partial n$ does not vanish whenever $\left.\partial \psi_{n} / \partial n(x)=0\right)$.

(3) The nodal set $N=N\left(\psi_{n}\right)$ is the finite union of non-intersecting smooth hyper-surfaces

$$
N=\left(\bigcup_{k} C_{k}\right) \cup\left(\bigcup_{l} B_{l}\right)
$$

(see Fig. 1), where

(4) Each $C_{k}$ is a closed smooth hyper-surface in $\Omega$.

(5) Each $B_{l}$ is a smooth hyper-surface, whose boundary lies in $\partial \Omega ; B_{l}$ intersects $\partial \Omega$ transversally.

In fact, the intersections of $B_{l}$ with $\partial \Omega$ are orthogonal, but we will not need to use this information.

We will be dealing with the generic situation only, in the sense that all conditions (1)-(5) above are satisfied for the eigenfunction in question. Partitions that we consider are required to satisfy similar assumptions. 
Definition 7. A partition $P=\left\{D_{j}\right\}$ of $\Omega$ will be called generic, if its nodal set $N=\Omega \backslash \bigcup D_{j}$ satisfies conditions (3)-(5) above.

We now briefly describe the constructions and results of the paper.

Given a generic $\nu$-partition $P$ and a sufficiently small positive number $\rho>$ 0 , we will introduce the set $\mathcal{P}_{\rho}$ of $\nu$-partitions that are "close" to $P$ in an appropriate sense. Here $\rho$ indicates a measure of closeness. The set $\mathcal{P}_{\rho}$ will be equipped with the structure of a real Hilbert manifold by identifying it with a ball in an appropriate functional Hilbert space. We will denote by $\mathcal{E}_{\rho}$ the subset of $\mathcal{P}_{\rho}$ that consists of equipartitions.

On the set $\mathcal{E}_{\rho}$ of equipartitions one can consider the functional

$$
\Lambda: \mathcal{E} \mapsto \mathbb{R}
$$

that maps a partition $P$ into the (common) lowest energy $\lambda_{1}\left(P_{j}\right)$ of any subdomain $P_{j}$. The notation $\Lambda$ does not contradict the one used previously in (3), since on equipartitions the two functionals obviously coincide. We will also need some other extensions of the functional $\Lambda$ from the set $\mathcal{E}_{\rho}$ of equipartitions to the whole $\mathcal{P}_{\rho}$. Let $c=\left(c_{1}, \ldots, c_{\nu}\right) \in \mathbb{R}^{\nu}$ be a unit simplex vector, i.e. such that $c_{j} \geq 0$ and $\sum c_{j}=1$. We define the functional $\Lambda_{c}$ on $\mathcal{P}_{\rho}$ as follows:

$$
\Lambda_{c}(P)=\sum c_{j} \lambda_{1}\left(P_{j}\right)
$$

where $P \in \mathcal{P}_{\rho}$ and $P_{j}$ are the sub-domains of this partition. It is obvious that for any unit simplex vector $c$ the restriction of $\Lambda_{c}$ to $\mathcal{E}_{\rho}$ coincides with $\Lambda$.

We will need the following auxiliary result:

\section{Proposition 8.}

(1) For any $c$, the functional $\Lambda_{c}$ on $\mathcal{P}_{\rho}$ is $C^{\infty}$-smooth.

(2) For a sufficiently small $\rho, \mathcal{E}_{\rho}$ is a smooth sub-manifold of $\mathcal{P}_{\rho}$ of codimension $\nu-1$.

(3) The functional $\Lambda$ on $\mathcal{E}_{\rho}$ is $C^{\infty}$-smooth.

This allows us now to formulate the first of the two main results of the paper:

Theorem 9. Let $P$ be a generic bipartite equipartition of a smooth domain $\Omega$. Then, the following statements are equivalent:

(1) $P$ is nodal (i.e., $P$ is the nodal partition of an eigenfunction $\psi$ of $H$ ).

(2) There exists a vector $c=\left(c_{1}, \ldots, c_{\nu}\right) \in \mathbb{R}^{\nu}, c_{j} \geq 0, \sum c_{j}=1$, such that $P$ is a critical point of the functional $\Lambda_{c}$ on $\mathcal{P}_{\rho}$. In this case,

$$
c_{j}=\|\psi\|_{L^{2}\left(P_{j}\right)}^{2}=\int_{P_{j}}|\psi(x)|^{2} d x .
$$

(3) $P$ is a critical point of the functional $\Lambda$ on $\mathcal{E}_{\rho}$.

If $\Omega$ is simply connected, the above statements are also equivalent to the following,

(4) At any of the boundary surfaces $C_{j}$ and $B_{l}$, the normal derivatives of the groundstates for the two adjacent sub-domains are proportional. 
Remark 10. If the domain $\Omega$ is simply connected, the partition graph of a generic partition is a tree and thus bipartite automatically. Indeed, if the partition graph is not a tree, we can remove at least one edge while keeping the graph connected. That means that $\Omega$ will also remain connected after removal of the corresponding piece of the partition boundary, $C_{j}$ or $B_{l}$ (call it $B$, for simplicity). However, the hyper-surface $B$ is either closed or has a boundary in $\partial \Omega$. Therefore, due to simply-connectedness of $\Omega$, its removal should disconnect $\Omega$.

Let $P$ be the partition corresponding to a generic eigenfunction. Theorem 9 implies that, up to corrections of higher order, the functional $\Lambda$ is given by

$$
\Lambda(P+\Delta P)=\Lambda(P)+F_{2}(\Delta P, \Delta P)+\text { higher order terms, }
$$

where $F_{2}$ - the Hessian - is a quadratic form on the tangent space of $\mathcal{E}_{\rho}$ at $P$ (which will be described in detail in Section 3). The Morse index of $P$ is then defined as the maximal dimension of the subspace on which $F_{2}$ is negative definite. Informally, the Morse index counts directions in which the function $\Lambda$ is unstable, i.e. decreasing in value. We also define the $\mu^{0}$ - index as the maximal dimension of a subspace on which $F_{2}$ is non-positive. If the Morse and $\mu^{0}$ indices coincide, the critical point is called non-degenerate.

Our second main result is the following interpretation of the nodal deficiency:

Theorem 11. Let $\psi_{n}$ be a generic (see Definition [6) eigenfunction of $H$ and $P$ be its nodal partition. Then $P$ is a non-degenerate critical point of $\Lambda$ restricted to $\mathcal{E}_{\rho}$ and the nodal deficiency $d_{n}=n-\nu_{\psi_{n}}$ is equal to the Morse index of $\Lambda$ at the point $P$.

Remark 12. To summarize Theorems 9 and 11, instead of looking at the minimal points of $\Lambda$, one has to look at the critical min-max points, where the maximum is taken over a subspace of dimension equal to the nodal deficiency $d_{n}$. This explains, in particular, why the minimal partitions correspond the Courant sharp eigenfunctions only.

The structure of this article is as follows. Section 2 contains a brief exposition of the well known Rayleigh-Hadamard formula for the derivative of an eigenvalue with respect to the domain variation. The manifolds $\mathcal{P}_{\rho}$ and $\mathcal{E}_{\rho}$ are introduced in Section 3, where also the Proposition 8 is proven. Theorems 9] and 11 are proven in sections 4 and 5. Section 6 contains final remarks and conclusions. In particular, it offers various possible generalizations of the results of this paper.

\section{DOMAin VARIATION FORMUlas}

In this section we provide the formulas for eigenvalue perturbation due to domain variation, which will be important for our considerations. Such formulas have a long history, going back to J. Rayleigh [50] and J. Hadamard [29] and are still being developed (see [20, 22, 24, 25, 28, 33, 36, 39, 48] for further results and references).

Let $D$ be a proper sub-domain of $\Omega$ (i.e., the closure of $D$ belongs to $\Omega$ ) with a smooth boundary $C=\partial D$. Later on in this text $D$ will be one of 


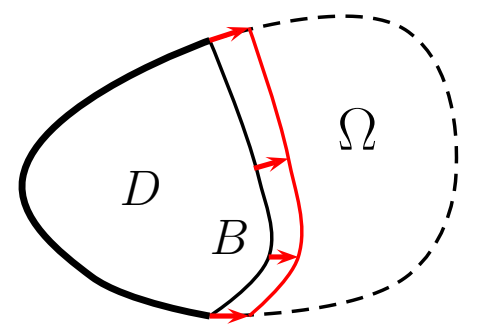

Figure 2. Domain $D$ cut out from $\Omega$ by $B$ and its deformation.

the sub-domains of a partition. We denote by $\psi_{1}(D)$ and $\lambda_{1}(D)$ the positive normalized groundstate and the corresponding eigenvalue of $H(D)$.

We are interested in the variation of $\lambda_{1}(D)$ with respect to infinitesimal smooth deformations of the boundary $C$. To make it precise, let us consider the unit external normal vector field on $C$ and extend it into a neighborhood $U$ of $C$ to a smooth unit length vector field $N(x)$ whose trajectories are the normals to $C$. Let us now also have a sufficiently smooth real valued function $f(x)$ in $U$. Consider the normal to $C$ vector field $f(x) N(x)$ and the corresponding evolution operators $G_{t}$ of the "time" $t$ shift along the trajectories of this field. They are defined for sufficiently small values of $t$ and produce deformed surfaces $C_{t}=G_{t} C$ and the variable domains $D_{t}$ bounded by these surfaces. Correspondingly, the ground state eigenvalue $\lambda_{1}\left(D_{t}\right)$ is a function of $t$. The following result (Rayleigh-Hadamard formula) is well known (e.g. $20,22,24,25,28,29,36,39,48,50$ ) for the case when $V(x)=0$. However, its proof (e.g. the one in [28]) is valid for non-zero potentials as well.

Theorem 13. The $t$-derivative at $t=0$ of the eigenvalue $\lambda_{1}\left(D_{t}\right)$ is given by the formula

$$
\lambda_{1}^{\prime}=-\int_{C}\left(\frac{\partial \psi_{1}(D)}{\partial n}\right)^{2} f(x) d S,
$$

where $\partial / \partial n$ denotes the external normal derivative on $C$ and $\psi_{1}(D)$ is, as before, the normalized Dirichlet ground-state of the domain D.

Remark 14. If one uses a smooth vector field $M(x)$ instead of the unit normal vector field $N(x)$, an analog of formula (44) for the $t$-derivative at $t=0$ of the eigenvalue $\lambda_{1}\left(D_{t}\right)$ is given by

$$
\lambda_{1}^{\prime}=-\int_{C}\left(\frac{\partial \psi_{1}(D)}{\partial n}\right)^{2} f(x) M(x) \cdot N(x) d S,
$$

where $\partial / \partial n$ denotes the external normal derivative on $C$ and $M \cdot N$ is the inner product of vectors $M$ and $N$.

Some of the nodal sub-domains will reach the boundary. We thus also need to consider the case of $D \subset \Omega$ that is cut out from $\Omega$ by a smooth surface (curve when $d=2$ ) $B$ transversal to the boundary $\partial \Omega$ (Fig. 2). 
Consider again the external unit normal vector field $N(x)$ to $B$. We can assume that this field is modified near the boundary $\partial \Omega$ to a smooth vector field $M(x)$ that is (i) non-tangential to $B$ and (ii) tangential to $\partial \Omega$ on the intersection $B \cap \partial \Omega$. It can then be extended to a smooth vector field $M$ near $B$ such that the trajectories that start at the boundary points (i.e., points of $B \cap \partial \Omega$ ) stay on the boundary $\partial \Omega$ and such that the level sets $G_{t} B$ for small values of $t$ are transversal to $\partial \Omega$. Let $f \in H^{s}(B)$ (for a sufficiently large $s$ ) be a real valued function on $B$. Then, similarly to the case of an internal part of $\Omega$ considered above, one can deform the surface $B$ by a vector field $f M$, which will define sub-domains $D_{t}$ of $\Omega$ with their boundaries $B_{t}$ transversal to $\partial \Omega$. With this setup, the variation formula completely identical to (5) holds.

Although in this case the variation formula may or may not have been written before, the proof of Theorem 13 given, for instance, in [28] carries through without any change.

Remark 15. If the boundary of the domain $D$ is not connected, then the same variation formula holds, involving the sum of integrals over each connected component $C_{j}$ and $B_{l}$ of the boundary.

\section{Manifolds of partitions. Proof of Proposition 8}

Let us now consider a generic $\nu$-partition $P=\left\{P_{j}\right\}_{j=1}^{\nu}$ of $\Omega$. We need to introduce a manifold structure into the space of "nearby" $\nu$-partitions. The previous section suggests a simple way of doing so. Namely, let $\left\{C_{k}, B_{l}\right\}$ be the smooth connected surfaces constituting the boundaries between the subdomains $P_{j}$ inside $\Omega$ ( $\partial \Omega$ also contributes to the boundaries of some of the subdomains, but is not taken into account, since it is not going to be changed). Let us fix a smooth non-tangential to $\left\{C_{k}, B_{l}\right\}$ vector field $M$ in a neighborhood $U$ of $\left(\cup_{k} C_{k}\right) \cup\left(\cup_{l} B_{l}\right)$, which satisfies the conditions imposed in the previous section (e.g., one can assume that outside of a neighborhood of $\partial \Omega$, this is just the unit normal field $N(x)$, which is smoothly modified near $\partial \Omega$ to be tangential to $\partial \Omega$ ). Such a field always exists under the genericity condition imposed on the partition $P$.

Let us pick a sufficiently large positive number $s$ (e.g., $s>(d+4) / 2$ will suffice) and consider the space

$$
\mathcal{F}:=\left(\oplus_{k} H^{s}\left(C_{k}\right)\right) \bigoplus\left(\oplus_{l} H^{s}\left(B_{l}\right)\right),
$$

where $H^{s}$ is the standard Sobolev space of order $s$. We also introduce a continuous linear extension operator

$$
E: \mathcal{F} \rightarrow H^{s+1 / 2}(U)
$$

In other words, the restriction of $E(f)$ to $C_{k}$ coincides with $f$. Such an extension operator is well known to exist (see, e.g. 41]). We will also assume that all the extended functions $E(f)$ vanish outside of a small neighborhood of the nodal set, which can be achieved by multiplication by an appropriate smooth cut-off function.

Consider the ball $\mathcal{B}_{\rho}$ of a small radius $\rho>0$ around the origin in the space $\mathcal{F}$. Let $f \in \mathcal{B}_{\rho}$ and $G_{f}$ be the shift by time $t=1$ along the trajectories 
of the vector field $E(f)(x) M(x)$. For a sufficiently small $\rho>0, G_{f}$ is a diffeomorphism, which preserves the boundary $\partial \Omega$. Its action on the surfaces $C_{k}$ and $B_{l}$ leads to another $\nu$-partition $G_{f} P$ of $\Omega$, which is close to the original partition $P$. We will denote this set of partitions by $\mathcal{P}_{\rho}$ and identify it with the ball $\mathcal{B}_{\rho}$ in the Hilbert space $\mathcal{F}$. This, in particular, introduces the structure of a Hilbert manifold on $\mathcal{P}_{\rho}$. Notice also that we will use consistent numbering of the sub-domains of partitions in $\mathcal{P}_{\rho}$. Namely,

$$
\left(G_{f} P\right)_{j}=G_{f}\left(P_{j}\right),
$$

where, as before, for a partition $\Pi$ we denote by $\Pi_{j}$ its $j$ th sub-domain, and $P_{j}$ are the sub-domains of the original (unperturbed) partition $P$.

We introduce now a mapping $\Xi$ from $\mathcal{P}_{\rho}$ into $\mathbb{R}^{\nu}$ as follows: for a partition $\Pi$ we define

$$
\Xi(\Pi):=\left(\lambda_{1}\left(\Pi_{1}\right), \lambda_{1}\left(\Pi_{2}\right), \ldots, \lambda_{1}\left(\Pi_{\nu}\right)\right) .
$$

Let

$$
\Delta:=(\lambda, \lambda, \ldots, \lambda)
$$

be the diagonal in $\mathbb{R}^{\nu}$.

Definition 16. The set $\mathcal{E}_{\rho}$ consists of all equipartitions in $\mathcal{P}_{\rho}$, i.e. $\Pi \in \mathcal{P}_{\rho}$ satisfying

$$
\lambda_{1}\left(\Pi_{1}\right)=\lambda_{1}\left(\Pi_{2}\right)=\cdots=\lambda_{1}\left(\Pi_{\nu}\right) .
$$

To put it differently,

$$
\mathcal{E}_{\rho}=\Xi^{-1}(\Delta)
$$

One notices that the restriction of the mapping $\Xi$ to $\mathcal{E}_{\rho}$ is essentially the functional $\Lambda$ of the Introduction. More precisely,

$$
\Xi(\Pi)=(\Lambda(\Pi), \ldots, \Lambda(\Pi)) .
$$

We are ready now to prove the Proposition 8 .

3.1. Proof of the Proposition 8. We prove first the following auxiliary result, which immediately implies the first statement of the Proposition 8 .

Lemma 17. For a small $\rho>0$, the mapping $\Xi: \mathcal{P}_{\rho} \rightarrow \mathbb{R}^{\nu}$ is $C^{\infty}$.

Proof. It is sufficient to prove that for any $j$, the mapping

$$
f \rightarrow \lambda_{1}\left(G_{f}\left(P_{j}\right)\right)
$$

is smooth as a mapping from the ball $\mathcal{B}_{\rho}$ to $\mathbb{R}$, if $\rho$ is sufficiently small. Thus, one can restrict attention to a single sub-domain $D$. The often employed in such circumstances idea is to replace domain dependence with varying the coefficients of the differential operator in a fixed domain. Then the smooth dependence of $\lambda_{1}$ becomes a standard perturbation theory result (e.g., [25, 34]).

Let us consider first the case of a sub-domain $D$ that does not touch the boundary. Consider the mapping

$$
\Phi_{f}: x \rightarrow y:=x+E(f)(x) M(x)
$$


of the domain $D$ into $\Omega$. For sufficiently small $\rho>0$, it is a $C^{2}$-diffeomorphism of $D$ onto a "nearby" sub-domain $D^{*}$ of $\Omega$. The quadratic form of the operator $H\left(D^{*}\right)$ is given as

$$
\int_{D^{*}}\left(\left|\frac{\partial u}{\partial y}\right|^{2}+V(y)|u(y)|^{2}\right) d y .
$$

Changing variables back from $y$ to $x$, we arrive at an operator $H_{f}(D)$ in the fixed spatial domain $D$, but with variable coefficients now:

$$
\left(H_{f} u\right)(x)=\nabla \cdot A_{f}(x) \nabla u(x)+V_{f}(x) u(x),
$$

where the matrix-valued function $A_{f}(x)$ is of class $C^{1}, f \rightarrow A_{f}$ is a $C^{\infty}$ mapping from $H^{s}$ to the space of $C^{1}$-matrix functions, and $f \rightarrow V_{f}$ is a $C^{\infty}$ mapping from $H^{s}$ to $C^{2}$.

We have thus replaced the domain dependence with the smooth dependence of the coefficients of the operator. The operator $H_{f}$ acts continuously from $H^{2}(\Omega)$ to $L^{2}(\Omega)$ and for $f=0$ coincides with $H(D)$. Moreover, the mapping $A_{f} \rightarrow H_{f}$ is a continuous linear mapping from the space of $C^{1}$-matrix functions to the space of bounded operators from $H^{2}(\Omega)$ to $L^{2}(\Omega)$. Thus, for a sufficiently small $\rho$ we get a smooth family of Fredholm operators between the aforementioned spaces. Due to the simplicity of $\lambda_{1}(D)$, the standard perturbation theory shows that $\lambda_{1}\left(H_{f}\right)$ depends smoothly on $f$, for a sufficiently small radius $\rho$.

A similar consideration works when $D$ reaches the boundary of $\Omega$, i.e. at least one of the boundaries $B_{l}$ is involved. Without loss of generality, we can assume that only one such $B_{l}$ is involved. Introducing an appropriate smooth coordinate change, one can reduce consideration to the cylinder $B \times(-\varepsilon, \varepsilon)$ for small $\varepsilon>0$, with $\partial B \times(-\varepsilon, \varepsilon)$ as the corresponding part of $\partial \Omega$. Then the same reduction to a fixed domain but varying operator as before is possible, which again implies smooth dependence of $\lambda_{1}$ on $f$.

Let us now address the second statement of the Proposition, that $\mathcal{E}_{\rho}$ is a smooth sub-manifold of $\mathcal{P}_{\rho}$ of co-dimension $\nu-1$. We will employ for this purpose the formula (9) in conjunction with the domain variation formula, equation (5), and a transversality theorem.

According to Lemma 17, the mapping $\Xi: \mathcal{P}_{\rho} \rightarrow \mathbb{R}^{\nu}$ is a smooth mapping of Banach manifolds. The pre-image of the diagonal $\Delta \subset \mathbb{R}^{\nu}$ coincides, according to (9), with $\mathcal{E}_{\rho}$. We would like to know whether this pre-image is a smooth sub-manifold, and of what co-dimension. This is exactly the question tackled by the transversality theorems. Namely, if we can show that the mapping $\Xi$ is transversal to the diagonal one-dimensional sub-manifold $\Delta$ of $\mathbb{R}^{\nu}$, this will prove that the pre-image of $\Delta$ is a smooth sub-manifold of co-dimension $\nu-1$.

Definition 18. (e.g., [1,9,40]) The mapping $\Xi: \mathcal{P}_{\rho} \rightarrow \mathbb{R}^{\nu}$ is transversal to $\Delta$, if at any point $\zeta=\Xi(v), v \in \mathcal{P}_{\rho}$, that belongs to $\Delta$, the vector sum of the tangent space $T_{\zeta} \Delta$ to $\Delta$ at $\zeta$ and of the range $D \Xi\left(T_{v} \mathcal{P}_{\rho}\right)$ of the differential $D \Xi$ on the tangent space $T_{v} \mathcal{P}_{\rho}$ is the whole space $\mathbb{R}^{\nu}$.

Theorem 19. (e.g., [1, Sect. 3, Theorem 2] or [9, Sect. 5.11.7]) If $\Xi$ is transversal to $\Delta$, then $\mathcal{E}_{\rho}=\Xi^{-1}(\Delta)$ is a smooth sub-manifold of $\mathcal{P}_{\rho}$ of co-dimension $\nu-1$. 
Thus, to finish the proof of the second statement of Proposition 8, it only remains to prove the transversality of the mapping $\Xi$ to the diagonal $\Delta$. The Rayleigh-Hadamard domain variation formulas are helpful here.

The tangent space to the diagonal is spanned by the vector $(1,1, \ldots, 1)$. We will demonstrate that the range of $D \Xi$ contains a subspace of dimension at least $\nu-1$. If the dimension is $\nu$, there is nothing further to prove, but if the dimension is $\nu-1$ we will show that the vector $(1,1, \ldots, 1)$ does not belong to the subspace.

Consider the partition graph $\Gamma$ that corresponds to an (automatically generic) $\nu$-partition $P \in \mathcal{P}_{\rho}$. The vertices of the graph correspond to the sub-domains $P_{j}$ and the edges to the interfaces $C_{j}, B_{l}$. We will identify the target space $\mathbb{R}^{\nu}$ of the mapping $\Xi$ with the space of real valued functions on the set $V$ of vertices of the graph $\Gamma$. Consider a pair of adjacent sub-domains $P_{i}$ and $P_{j}$ with the common part of their boundary $S$ (one of $C_{j}, B_{l}$ ). We consider the corresponding vertices $v_{i}, v_{j}$ and the edge $s$ of $\Gamma$. We restrict our attention to functions $f \in \mathcal{F}$ that are non-zero on $S$ only and find the corresponding directional (Gâteaux) derivative of $\Xi$ at $P$ in the direction of $f$. Equation (5) shows that the only non-zero components of this derivative correspond to vertices $v_{i}$ and $v_{j}$. Since $M(x)$ is non-tangential to $S, M(x) \cdot N(x)$ is sign-definite. Choosing $f$ of the same sign we get

$$
D_{f} \Xi_{i}=-\int_{S}\left(\frac{\partial \psi_{1}\left(P_{i}\right)}{\partial n}\right)^{2} f(x) M(x) \cdot N(x) d x<0
$$

and, similarly, $D_{f} \Xi_{j}>0$. Here we assumed that the normal $N(x)$ is directed outward with respect to $P_{i}$ and, correspondingly, inward with respect to $P_{j}$. We also used the fact that, due to the standard uniqueness theorems, $\partial \psi_{1}\left(P_{i}\right) / \partial n$ is not everywhere zero.

Repeating this procedure for every pair of adjacent sub-domains we arrive at a collection of vectors, one for each edge of the partition graph, that have two non-zero components of the opposite sign each. To characterize the space spanned by these vectors, we arrange them as rows of a matrix and find its kernel. Due to the connectedness of the graph, the kernel is at most onedimensional. If the kernel is empty, the vectors we found span all of $\mathbb{R}^{\nu}$. If the kernel is spanned by a vector $u$ (this is the case if the domain $\Omega$ is simply connected, as this implies that the partition graph is a tree), then $u$ must have all components of the same sign. The vector $u$ is therefore not orthogonal to the vector $(1,1, \ldots, 1)$, and the latter vector complements the derivative vectors to span $\mathbb{R}^{\nu}$.

This finishes the proof of transversality and thus of the second statement of the proposition. Since the first two claims of the proposition imply the third one, the proof is completed.

\section{Proof of Theorem 9}

4.1. Proof of the equivalence (11) $\Leftrightarrow$ (2). If $P$ is the nodal partition of a real-valued eigenfunction $\psi$, then, as we have already mentioned before (Proposition (3), the restrictions of $\psi$ to the nodal domains are proportional to the groundstates in these domains. Denote these proportionality constants by 
$a_{j}$. Since the eigenfunction $\psi$ is continuously differentiable, the groundstates $\psi_{1}\left(P_{j}\right)$ scaled with the corresponding factors $a_{j}$ have matching normal derivatives at the common boundaries:

$$
\left.a_{i} \frac{\partial \psi_{1}\left(P_{i}\right)}{\partial n}\right|_{S}=\left.a_{j} \frac{\partial \psi_{1}\left(P_{j}\right)}{\partial n}\right|_{S}
$$

where sub-domains $P_{i}$ and $P_{j}$ have the common boundary $S$, and $n$ is a normal vector to $S$.

Now let $c_{k}=a_{k}^{2}$ and consider the Gâteaux derivative of the functional $\Lambda_{c}$ in the direction $f$ that is non-zero only on a single boundary between the sub-domains $P_{i}$ and $P_{j}$. Since the only affected terms in $\Lambda_{c}$ are $a_{i}^{2} \lambda\left(P_{i}\right)$ and $a_{j}^{2} \lambda\left(P_{j}\right)$, the derivative is

$$
\int_{S}\left(a_{j}^{2}\left(\frac{\partial \psi_{1}\left(P_{j}\right)}{\partial n}\right)^{2}-a_{i}^{2}\left(\frac{\partial \psi_{1}\left(P_{i}\right)}{\partial n}\right)^{2}\right) f(x) M(x) \cdot N(x) d S,
$$

where we applied the Rayleigh-Hadamard formula, equation (15). The difference in signs arises since $N(x)$ points outward with respect to $P_{i}$ but inward with respect to $P_{j}$. Now we observe that, due to (10), the integrand is identically equal to zero and thus the Gâteaux derivative in the direction of $f$ is equal to zero. The same is obviously true for arbitrary variations $f$, involving any number of boundaries. Thus, the nodal partition is a critical point of the functional $\Lambda_{c}$.

Conversely, if a partition $P_{\rho}$ is a critical point of $\Lambda_{c}$, we get that the Gâteaux derivative of $\Lambda_{c}$ is zero in any direction $f(x)$. This implies the equality

$$
c_{i}\left(\frac{\partial \psi_{1}\left(P_{i}\right)}{\partial n}\right)^{2}=c_{j}\left(\frac{\partial \psi_{1}\left(P_{j}\right)}{\partial n}\right)^{2}
$$

on the common boundary $S$ of any two neighboring domains $P_{i}$ and $P_{j}$. Setting $\alpha_{k}= \pm \sqrt{c_{k}}$ and choosing the signs so that any two neighboring domains have different signs (possible due to bipartiteness) ensures that (10) is satisfied. Then the function $\psi$ defined by

$$
\left.\psi\right|_{P_{k}}=a_{k} \psi_{1}\left(P_{k}\right)
$$

is an eigenstate of $H$.

4.2. Proof of the equivalence (2) $\Leftrightarrow(\underline{3)})$. If $P$ is a critical point on $\mathcal{P}_{\rho}$ of the functional $\Lambda_{c}$, then the restriction of $\Lambda_{c}$ to $\mathcal{E}_{\rho}$ is a critical point on $\mathcal{E}_{\rho}$. But on $\mathcal{E}_{\rho}$ any functional $\Lambda_{c}$ coincides with $\Lambda$.

Conversely, assume that $P$ is a critical point of $\Lambda$ on $\mathcal{E}_{\rho}$. We can extend the functional $\Lambda$ to the whole $\mathcal{P}_{\rho}$ as $\lambda_{1}\left(P_{1}\right)$. Since $\mathcal{E}_{\rho}$ can be given by the smooth relations $\lambda_{1}\left(P_{1}\right)-\lambda_{1}\left(P_{j}\right)=0$ for $j=2,3, \ldots, \nu$, the Lagrange multiplier method implies that $P$ must be a critical point of a non-trivial linear combination $\Lambda_{b}:=\sum b_{j} \lambda_{1}\left(P_{j}\right)$. All $b_{j}$ are of the same sign: otherwise there are two neighboring domains with $b_{j}$ of different signs and the variation of $\Lambda_{b}$ with respect to the boundary between the two domains cannot be zero for a sign-definite $f(x)$ (see equation (11)). Thus the vector of coefficients $b_{j}$ can be normalized to be a unit simplex vector. This finishes the proof of Theorem 9 , 


\section{Proof of Theorem 11}

Let us present first the strategy of the proof. As we have already seen, it is sometimes useful to play with different extensions of the functional $\Lambda$ from the (local patch of the) space $\mathcal{E}_{\rho}$ of equipartitions to a larger manifold. While previously it was the (local patch of the) space of all partitions, now we need some further extension. Indeed, we would like to compare somehow the nodal count $\nu$ (which is fixed) with the consecutive number $n$ of an eigenfunction $\psi_{n}$. It is hard to observe where the information about $n$ is hidden in the spaces of partitions themselves. On the other hand, the quadratic form (or Rayleigh quotient) contains this information. To use it we will extend the functional $\Lambda$ to a larger space that is a functional space, not just a set of domain partitions. Then we will have to restrict back in order to compare the Morse indices of $\Lambda$ and of its extension.

Before implementing this program, in the following sub-sections we start proving some auxiliary statements that will come handy later on.

\subsection{Critical points on direct sums of spaces.}

Theorem 20. Let $X=Y \bigoplus Y^{\prime}$ be a direct decomposition of a Banach space. Let also $f: X \rightarrow \mathbb{R}$ be a smooth functional such that $(0,0) \in X$ is its critical point of Morse index $m$.

If for any $y$ in a neighborhood of zero in $Y$, the point $(y, 0)$ is a critical point of $f$ over the affine subspace $\{y\} \times Y^{\prime}$, then the Hessian $F_{2}$ of $f$ at the origin, as a quadratic form in $X$, is reduced by the decomposition $X=Y \bigoplus Y^{\prime}$.

In particular, let $Y$ be the locus of minima of $f$ over the affine subspaces $\{y\} \times Y^{\prime}$, i.e.

$$
(y, 0)=\arg \min _{y^{\prime} \in Y^{\prime}} f\left(y, y^{\prime}\right),
$$

for any $y$ in a neighborhood of zero in $Y$. Then the Morse index of 0 as a critical point of the restriction $\left.f\right|_{Y}$ is equal to $m$ (i.e., the same as the Morse index of this point on the whole neighborhood of zero in $X$ ).

Finally, if $(0,0)$ is a non-degenerate critical point of $f$ on $X$, then 0 is non-degenerate as a critical point of $\left.f\right|_{Y}$.

Proof. We can assume, without loss of generality, that $f(0,0)=0$. Using this and the condition of the criticality of the origin, the Taylor formula of the second order for $f$ on $X$ near the origin is

$$
f\left(y, y^{\prime}\right)=A(y, y)+B\left(y^{\prime}, y^{\prime}\right)+C\left(y, y^{\prime}\right)+\text { higher order terms. }
$$

Here $A$ is a quadratic form in $Y, B$ is a quadratic form in $Y^{\prime}$ and $C$ is a bilinear form acting on $Y \times Y^{\prime}$. We will now take the Gâteaux derivative of $f\left(y, y^{\prime}\right)$ in the direction $z^{\prime} \in Y^{\prime}$ and evaluate it at $(y, 0)$.

The derivative of $A(y, y)$ is zero (since $A$ does not depend on $y^{\prime}$ ). The derivative of $B\left(y^{\prime}, y^{\prime}\right)$ is $B\left(z^{\prime}, y^{\prime}\right)+B\left(y^{\prime}, z^{\prime}\right)$, which vanishes after substituting $y^{\prime}=0$. Thus we find

$$
D_{\left(0, z^{\prime}\right)} f(y, 0)=C\left(y, z^{\prime}\right)
$$

which must be zero since $(y, 0)$ is a critical point over $\{y\} \times Y^{\prime}$. Since $z^{\prime}$ and $y$ are arbitrary (provided $y$ is sufficiently close to 0 ), the bilinear form $C$ is 
identically zero. The Hessian is thus

$$
F_{2}=A(y, y)+B\left(y^{\prime}, y^{\prime}\right)
$$

This proves the first statement of the Theorem.

If condition (12) is fulfilled, then $B\left(y^{\prime}, y^{\prime}\right)$ is non-negative definite and the negative subspaces of $F_{2}$ and $A$ coincide (after the natural projection).

Finally, if $(0,0)$ is non-degenerate, it implies that $\mu^{0}(A)=\mu(A)$, where $\mu$ denotes the Morse index. Indeed, otherwise $\mu^{0}\left(F_{2}\right) \geq \mu^{0}(A)>\mu(A)=\mu\left(F_{2}\right)$ which contradicts the non-degeneracy of $(0,0)$.

Remark 21. It is obvious from the proof of the theorem that its second statement can be generalized in the following manner: if $Y$ is the locus of critical points of $f$ of index $m^{\prime}$ (locally independent of $y$ ) then the Morse index of the restriction $\left.f\right|_{Y}$ is equal to $m-m^{\prime}$. We will only need $m^{\prime}=0$ in the present manuscript but the more general version becomes useful in other contexts, such as [7].

5.2. Some objects needed for the proof. Due to the local character of Theorem 11, all the constructions below are needed only locally, near a generic eigenfunction $\psi_{n}$ indicated in the statement of the theorem, and correspondingly near its nodal partition. The basic notions and facts concerning finite- or infinite- dimensional vector-bundles that we use below can be found in many standard sources on topology (e.g., [4,35]) or in the survey [52], where such bundles are studied in relation to the operator theory.

We will be considering again the (local) manifold $\mathcal{P}_{\rho}$ of partitions "close" to the nodal partition $P\left(\psi_{n}\right)$ and its sub-manifold $\mathcal{E}_{\rho}$ of codimension $\nu-1$ that consists of equipartitions only.

Definition 22. In the trivial bundle $\mathcal{P}_{\rho} \times H_{0}^{1}(\Omega)$ over $\mathcal{P}_{\rho}$, we consider a fibered subset $B$ that has the fiber over a partition $P$ consisting only of functions vanishing on the partition's interfaces $N \cup \partial \Omega$. In other words, this fiber is $H_{P}^{1}:=\bigoplus_{j} H_{0}^{1}\left(P_{j}\right)$.

Lemma 23. $B$ is a smooth locally trivial vector sub-bundle of the trivial bundle

$$
\mathcal{P}_{\rho} \times H_{0}^{1}(\Omega) \mapsto \mathcal{P}_{\rho}
$$

Proof. The proof follows the same line as the one of Lemma 17. Namely, the dependence on the partition $P$ is replaced, using a smooth family of diffeomorphisms, with a fixed partition $\Pi$. The corresponding change of variables in the functions from $H_{P}^{1}$ maps them to $H_{\Pi}^{1}$. Then $B$ becomes just the trivial subbundle $\mathcal{P}_{\rho} \times\left(\bigoplus_{j} H_{0}^{1}\left(\Pi_{j}\right)\right)$ in $\mathcal{P}_{\rho} \times H_{0}^{1}(\Omega) \mapsto \mathcal{P}_{\rho}$. Inverting the diffeomorphisms provides a smooth trivialization of $B$, which proves the lemma.

Definition 24. We denote by $S B$ the locally-trivial bundle of the unit (in $L_{2}$-norm) spheres of the fibers of $B$.

The restrictions of $B$ and $S B$ to $\mathcal{E}_{\rho}$ (clearly locally-trivial bundles) will be denoted by $B_{E}$ and $S B_{E}$ correspondingly.

We will now restrict the bundle $B$ further. 
Definition 25. We denote by $C$ the vector bundle whose fiber over a partition $P$ consists of functions of the form $\sum_{j} c_{j} \psi_{1}\left(P_{j}\right)$, where $c_{j}$ are real constants and $\psi_{1}\left(P_{j}\right)$ is the normalized positive groundstate on the sub-domain $P_{j}$.

Correspondingly, $S C$ consists of the unit (in $L_{2}$-norm) spheres of $C$ and $C_{E}$ and $S C_{E}$ are restrictions of the corresponding fibered sets to $\mathcal{E}_{\rho}$.

The following lemma shows that $C$ and $S C$ are locally-trivial bundles, and, in particular, $C$ is a locally trivial vector bundle.

Lemma 26. $C$ is a smooth $\nu$-dimensional vector sub-bundle of $B$, and thus of the trivial bundle

$$
\mathcal{P}_{\rho} \times H_{0}^{1}(\Omega) \mapsto \mathcal{P}_{\rho}
$$

Proof. The proof follows the same line as in Lemmas 17 and 23. Namely, after applying a smooth family of diffeomorphisms, one deals with a fixed partition $\Pi$, but instead with the operator whose coefficients depend smoothly on $P$. Perturbation theory shows that the corresponding ground-state $f_{j}$ in each sub-domain $\Pi_{j}$ depends smoothly on $P$, as a vector in $H_{0}^{1}\left(\Pi_{j}\right)$. We extend it, without changing the notation, by zero to the whole domain $\Omega$. Then $\left\{f_{j}\right\}$ is a smoothly dependent on $P$ frame of $\nu$ linearly independent vectors in $H_{0}^{1}(\Omega)$. Thus, this frame spans a smooth finite-dimensional vector-bundle. After applying the inverses of the diffeomorphisms, we get the claim of the lemma.

5.3. The functional $\Lambda$ and the quadratic form of $H$. Consider the quadratic form on $H_{0}^{1}(\Omega)$

$$
Q[f]:=\int_{\Omega}\left(|\nabla f(x)|^{2}+V(x)|f(x)|^{2}\right) d x .
$$

It can, by restricting to each fiber, be defined as a smooth functional on the vector bundle $B$ and its sub-bundles that we considered above.

Lemma 27. The point $\left(P\left(\psi_{n}\right), \psi_{n}\right)$ in $S B$ is a non-degenerate critical point of $Q$ of Morse index $\mu=n-1$.

Proof. It is clear that $Q$ on $H_{0}^{1}(\Omega)$ has an $n$-1-dimensional subspace on which its Hessian at $\psi_{n}$ is negative. Namely, this is the subspace generated by the eigenfunctions $\psi_{1}, \ldots, \psi_{n-1}$. If we show that these directions are among the tangential ones to $S B$ at $\left(P\left(\psi_{n}\right), \psi_{n}\right)$, this will prove that $\mu \geq n-1$.

Due to the locally-trivial structure of $S B$, there are two main ways to get tangential vectors to $S B$. One is to vary the partition $P$ (which will give "horizontal" tangent vectors to the bundle $S B$ ). The other is to keep the partition fixed, while varying the function $\psi_{n}$, keeping the nodal set fixed ("vertical" tangent vectors). The vertical tangent vectors are just arbitrary functions in $H_{0}^{1}(\Omega)$ that vanish on the nodal set $\mathcal{Z}$ of the partition $P\left(\psi_{n}\right)$ (we have previously denoted this space $\left.H_{P}^{1}\right)$. The horizontal tangential vectors look as follows:

$$
E(f) M \psi_{n}=E(f) N \psi_{n}(x)(M(x) \cdot N(x)),
$$

where $f \in H^{s}$ on the nodal set of $\psi_{n}$ is the function defining this set's infinitesimal variation. Also, the notation $X g$ for a vector field $X$ and a function 
$g$ (e.g., $M \psi_{n}$ and $N \psi_{n}$ ) mean the derivative of the function $g$ along the field $X$. Notice that, since $M(x)$ is non-tangential to $\mathcal{Z}, M(x) \cdot N(x)$ is a smooth separated from zero function on the nodal set.

We now show that any $\psi_{j}$ with $j<n$ can be represented as a sum of vertical and horizontal vectors. We notice that the genericity condition requires in particular that zero is a regular value of the normal derivative of $\psi_{n}$ on $\partial \Omega$. This implies that the derivative $N \psi_{n}$ is a smooth function that has a nondegenerate zero at $\partial \Omega$ on the nodal set $\mathcal{Z}$. Since $\psi_{j}$ is a smooth function vanishing on $\partial \Omega$, the function

$$
f(x):=\frac{\psi_{j}(x)}{\left(N \psi_{n}\right) M(x) \cdot N(x)}
$$

belongs to $H^{s}$ on the nodal set. Hence, the horizontal tangent vector in the direction of $f$

$$
h:=\left(N \psi_{n}\right) E(f) M(x) \cdot N(x)
$$

coincides with $\psi_{j}$ on the nodal set. This means that the difference $g:=\psi_{j}-h$ belongs to $H_{P}^{1}$, and thus is a vertical tangent vector. This shows that each eigenfunction $\psi_{1}, \ldots, \psi_{n-1}$ can be represented as the sum of a vertical and horizontal vectors and thus is tangent to $S B$. This proves the estimate $\mu \geq n-1$ for $S B$.

We will now prove that the index $\mu^{0}$ cannot exceed $n-1$. This will verify that $\mu=n-1$ and that the critical point is non-degenerate.

Suppose that there is an $n$-dimensional subspace $L$ in the tangent space to $S B$ at $\left(P\left(\psi_{n}\right), \psi_{n}\right)$, where the Hessian of $Q$ is non-positive. Since each fiber of $S B$ consists of functions from the space $H_{0}^{1}(\Omega)$, there is a tautological mapping $(P, f) \mapsto f$ from $S B$ into $H_{0}^{1}(\Omega)$ (in fact, into the set of functions of unit $L_{2}$-norm). If the Fréchet derivative of the tautological mapping has zero kernel, then the subspace $L$ will produce an $n$-dimensional subspace of $H_{0}^{1}(\Omega)$ transversal to $\psi_{n}$, where the Hessian of $Q$ at $\psi_{n}$ is non-positive, which is a contradiction. So, let us show that the kernel of the Fréchet derivative is zero. Due to the local trivial structure of $S B$, one sees that the image of any tangent vector under the Fréchet derivative has the form

$$
g=E(f)(x)\left(M \psi_{n}\right)+v(x),
$$

where the function $f \in \mathcal{F}$ is responsible for the infinitesimal variation of the nodal set of $\psi_{n}, E$ is the previously introduced extension operator from the nodal set to $\Omega$, and a function $v \in H_{P}^{1}(\Omega)$ corresponds to the infinitesimal variation in the fiber direction (i.e., the pair $(f, v)$ describes a tangent vector to $S B$ ). Suppose now that $g=0$. In particular, $\left.g\right|_{\mathcal{Z}}=0$. Taking into the account that $\left.v\right|_{\mathcal{Z}}=0$ (which is true for any function from the space $H_{P}^{1}$ ), we conclude that

$$
\left.E(f)(x)\left(M \psi_{n}\right)\right|_{\mathcal{Z}}=\left.f(x) M(x) \cdot \nabla \psi_{n}(x)\right|_{\mathcal{Z}}=0 .
$$

Since $\left.\nabla \psi_{n}(x)\right|_{\mathcal{Z}}$ is nonzero and orthogonal to $\mathcal{Z}$, and $M$ is transversal to $\mathcal{Z}$, we see that

$$
\left.M(x) \cdot \nabla \psi_{n}(x)\right|_{\mathcal{Z}} \neq 0 .
$$

This implies that $f(x)$ (defined on $\mathcal{Z}$ only) vanishes identically. Due to the linearity of the extension operator, the extension $E(f)$ vanishes everywhere in 
$\Omega$. Hence, $g=v$. Since $g=0$, we conclude that $v=0$ and thus the Fréchet derivative of the tautological mapping is injective. This finishes the proof of the lemma.

Lemma 28. The point $\left(P\left(\psi_{n}\right), \psi_{n}\right)$ in $S C$ is a non-degenerate critical point of $Q$ of Morse index $n-1$.

Proof. We know that the eigenfunction $\psi_{n}(x)$ has the form

$$
\psi_{n}(x)=\sum_{j=1}^{\nu} c_{j} \psi_{1}\left(D_{j}\right)(x)
$$

where $\left\{D_{j}\right\}$ is the nodal partition of $\psi_{n}$ and each ground state $\psi_{1}\left(D_{j}\right)$ is extended by zero to the whole domain $\Omega, \sum c_{j}^{2}=1$, and none of the coefficients $c_{j}$ are equal to zero. Thus the coefficients $c_{j}$ remain bounded away from zero in a neighborhood $U$ of $\psi_{n}$ in $S C$.

As we have already seen, $C$ is a smooth $\nu$-dimensional sub-bundle of $B$ and thus $S C$ is a smooth sub-manifold of $S B$. We will now introduce near the point $\left(P\left(\psi_{n}\right), \psi_{n}\right)$ a smooth foliation of $S B$ by manifolds transversal to $S C$, such that $S C$ will be the locus of minima of $Q$ over the leaves of this foliation. Then, after a smooth local change of coordinates, we will be in the situation of Theorem 20. Thus, the Morse index of $Q$ at $\left(P\left(\psi_{n}\right), \psi_{n}\right)$ on $S C$ will be equal to the one on $S B$, which will prove the first claim of the lemma.

So, let us finish the proof by constructing such a foliation. Consider the following mapping $\Upsilon$ from a neighborhood $U$ of $\left(P\left(\psi_{n}\right), \psi_{n}\right) \in S B$ to $\mathcal{P}_{\rho} \times S^{\nu-1}$, where $S^{\nu-1}$ is the unit sphere in $\mathbb{R}^{\nu}$ :

$$
\Upsilon(P, f)=\left(P,\|f\|_{L_{2}\left(P_{1}\right)}, \ldots,\|f\|_{L_{2}\left(P_{\nu}\right)}\right) .
$$

Notice that none of the components $\|f\|_{L_{2}\left(P_{j}\right)}$ of the vector $\Upsilon(P, f)$ vanishes (since this is the case for $\Upsilon\left(P\left(\psi_{n}\right), \psi_{n}\right)$ ).

By the arguments provided before, this is a smooth mapping. It is also clear that it is a submersion.

Let $w=\left(P, \sum c_{j} \psi_{1}\left(P_{j}\right)\right)$ be a point in $U \cap S C$ near $\left(P\left(\psi_{n}\right), \psi_{n}\right)$. Consider the leaf $L_{w}=\Upsilon^{-1}\left(P, c_{1}, c_{2}, \ldots, c_{\nu}\right)$. Due to the submersion property of $\Upsilon$, the leaves $L_{w}$ form near $\left(P\left(\psi_{n}\right), \psi_{n}\right)$ a smooth fibration. Since the differential of $\Upsilon$ on $S C$ is surjective, this foliation is transversal to $S C$. Moreover, the groundstate $\psi_{1}\left(P_{j}\right)$ is the location of the minimum of the corresponding Rayleigh quotient, and therefore

$$
Q[f]=\sum_{j=1}^{\nu} Q\left[\left.f\right|_{P_{j}}\right] \geq \sum_{j=1}^{\nu} Q\left[c_{j} \psi\left(P_{j}\right)\right], \quad c_{j}=\|f\|_{L_{2}\left(P_{j}\right)} .
$$

That is, the minimal value of $Q$ on the leaf $L_{w}$ is attained exactly when $f_{j}=$ $c_{j} \psi_{1}\left(P_{j}\right)$ for all $j$, i.e. on $S C$.

Lemma 29. The point $\left(P\left(\psi_{n}\right), \psi_{n}\right)$ is a (degenerate) critical point of $Q$ in $S C_{E}$ with the Morse index not less than $n-\nu$ and the $\mu^{0}$-index not more than $n-1$.

The restriction of the quadratic form $Q$ to the fibers of $S C_{E}$ is the pull-back of the functional $\Lambda$ from the base $\mathcal{E}_{\rho}$. In other words, if $\pi: S C_{E} \mapsto \mathcal{E}_{\rho}$ is the 
bundle projection, then for any $x \in S C_{E}$,

$$
Q(x)=\Lambda(\pi(x))
$$

Proof. According to Proposition 8, $S C_{E}$ is a smooth sub-manifold of $S C$ of co-dimension $\nu-1$. Hence, upon restriction to $S C_{E}$, an index (either Morse or $\mu^{0}$ ) cannot decrease by more than $\nu-1$. A restriction also can not increase an index. These observations, together with Lemma 28 , prove the first two claims.

Let $f=\sum_{j} c_{j} \psi_{1}\left(P_{j}\right)$ be an element of the fiber of $S C_{E}$ over a partition $P=\left\{P_{j}\right\}$. Then

$$
Q(f)=\sum\left|c_{j}\right|^{2} \lambda_{1}\left(P_{j}\right)
$$

Since $P$ is an equipartition, all the values $\lambda_{1}\left(P_{j}\right)$ are equal to the same value $\Lambda(P)$. Taking into account that $\sum\left|c_{j}\right|^{2}=\|f\|_{L_{2}(\Omega)}^{2}=1$, we get

$$
Q(f)=\sum\left|c_{j}\right|^{2} \lambda_{1}\left(P_{j}\right)=\Lambda(P)\left(\sum\left|c_{j}\right|^{2}\right)=\Lambda(P)
$$

which proves the last statement of the lemma.

5.4. Index of $\Lambda$ and nodal deficiency. We are now ready to prove Theorem 11 by obtaining estimates for the Morse index $\mu$ from two sides.

Proof of the estimate from below: $\mu \geq n-\nu_{\psi_{n}}$. From Lemma 29] we see that the pull-back of $\Lambda$ to $S C_{E}$ has Morse index at least $n-\nu$ at the point $\left(P\left(\psi_{n}\right), \psi_{n}\right)$, i.e. there is a tangent subspace of dimension at least $(n-\nu)$, where the Hessian of $Q$ is strictly negative definite. On the other hand, the pullback is constant along the fibers of $\pi: S C_{E} \mapsto \mathcal{E}_{\rho}$. Therefore the above subspace must correspond to such a subspace for the Hessian of $\Lambda$.

Proof of the estimate from above: $\mu \leq \mu^{0} \leq n-\nu_{\psi_{n}}$. We use Lemma29]again. It shows that the index $\mu^{0}$ of $\Lambda$ at $P\left(\psi_{n}\right)$ cannot exceed $(n-1)-(\nu-1)=n-\nu$. Indeed, the pullback of $\Lambda$ to $S C_{E}$ add a $(\nu-1)$-dimensional subspace on which the Hessian is zero. But the $\mu^{0}$-index on $S C_{E}$ is bounded by $n-1$.

To summarize, together with the trivial inequality $\mu \leq \mu^{0}$ (see the discussion prior to Theorem 11) we have shown that

$$
n-\nu_{\psi_{n}} \leq \mu \leq \mu^{0} \leq n-\nu_{\psi_{n}}
$$

This shows that the critical point is non-degenerate (i.e. $\mu=\mu^{0}$ ) and of Morse index $\mu=n-\nu_{\psi_{n}}$, finishing the proof of Theorem 11.

\section{FinAl REMARKS AND CONCLUSIONS}

(1) The results of this paper (Theorems 9 and 11) translate without any changes in their proofs to the case when $\Omega$ is a compact smooth Riemannian manifold with or without boundary.

(2) Smoothness conditions imposed on the domain, potential, and partition interfaces, can certainly be weakened. In this text, we have decided not to do so, in order not to complicate considerations unnecessarily. 
(3) The sets $\mathcal{P}$ and $\mathcal{E}$ that we considered involved only generic partitions, which allows perturbing the boundaries of sub-domains independently, simplifying the structure of the manifold of partitions and the consequent considerations of the text. In general, however, the smooth pieces of partition manifolds are joined into singular "varieties" $\mathcal{P}$ and $\mathcal{E}$, where the junctions occur when the partition interfaces start meeting each other. It would be interesting to see whether one could prove an analog of Theorems 9 and 11 for such non-generic partitions. The authors believe that something of this nature can be done.

(4) An interesting connection between the zeros of eigenfunctions and stability index with respect to another suitably defined perturbation has emerged recently. In the preprint [7] (see also [12] for an alternative proof), it was shown for the graph case that the "surplus" number of zeros $\phi_{n}-(n-1)$ is equal to the Morse index of the eigenvalue considered as a function of magnetic perturbation of the Hamiltonian. Here $\phi_{n}$ is the number of zeros of the $n$-th eigenfunction.

(5) The reader probably has noticed that there is a significant flexibility in our choice of the functional (e.g., Sobolev) spaces. Probably, one could achieve the same results working in the $C^{k}$ spaces of smooth functions. Our choice of the Sobolev scale was due to a known simpler interpretation of the Morse theory on Hilbert, rather than Banach, manifolds.

\section{ACKNOWLEDGMENTS}

The authors thank R. Band, Y. Colin de Verdiere, L. Friedlander, B. Helffer, H. Hezari, T. Hoffmann-Ostenhof, H. Raz, P. Sarnak, and S. Zelditch for useful discussions and references. We also are grateful to the reviewer for many important remarks. The work of G.B. was partially supported by the National Science Foundation grant DMS-0907968. The work of P.K. was partially supported by MSRI and IAMCS. The work of U.S. was partially supported by The Wales Institute of Mathematical and Computational Sciences (WIMCS), EPSRC (grant EP/G021287), and ISF (grant 166/09).

\section{REFERENCES}

[1] R. Abraham, Lectures of Smale on differential topology. Lecture Notes, Columbia Univ., New York, N.Y., 1962.

[2] J. H. Albert, "Nodal and critical sets for eigenfunctions of elliptic operators," in Partial differential equations (Proc. Sympos. Pure Math., Vol. XXIII, Univ. California, Berkeley, Calif., 1971), pp. 71-78, Providence, R.I.: Amer. Math. Soc., 1973.

[3] J. H. Albert, "Generic properties of eigenfunctions of elliptic partial differential operators," Trans. Amer. Math. Soc., vol. 238, pp. 341-354, 1978.

[4] M. F. Atiyah, "K-Theory", Benjamin, New York, 1967.

[5] R. Band, G. Berkolaiko, H. Raz, and U. Smilansky, "Critical partitions and nodal domains on quantum graphs," Comm. Math. Phys., 311, 815-838, 2012.

[6] G. Berkolaiko, H. Raz, and U. Smilansky, "Stability of nodal structures in graph eigenfunctions and its relation to the nodal domain count," J. Phys. A: Math. Theor. 45, 165203, 2012.

[7] G. Berkolaiko, Nodal count of graph eigenfunctions via magnetic perturbation, preprint arXiv:1110.5373. 
[8] G. Blum, S. Gnutzmann, and U. Smilansky. Nodal domains statistics: A criterion for quantum chaos. Phys. Rev. Lett., 88(11):114101, Mar 2002.

[9] N. Bourbaki, Éléments de mathématique. Fasc. XXXVI. Variétés différentielles et analytiques. Fascicule de résultats (Paragraphes 8 à 15). Actualités Scientifiques et Industrielles, No. 1347, Paris: Hermann, 1971.2006.

[10] J. Brüning, D. Klawonn, and C. Puhle. Comment on: "Resolving isospectral 'drums' by counting nodal domains" [J. Phys. A 38 (2005), no. 41, 8921-8933] by S. Gnutzmann, U. Smilansky and N. Sondergaard. J. Phys. A, 40(50):15143-15147, 2007.

[11] E. F. F. Chladni, Die Akustik, Brietkopf und Härtel, Leipzig 1802. French translation Traité d'Acoustique, Paris, Courcier 1809.

[12] Y. Colin de Verdière, Magnetic interpretation of the nodal defect on graphs, preprint arXiv:1201.1110

[13] M. Conti, S. Terracini, and G. Verzini, "On a class of optimal partition problems related to the Fučík spectrum and to the monotonicity formulae," Calc. Var. Partial Differential Equations, vol. 22, no. 1, pp. 45-72, 2005.

[14] R. Courant and D. Hilbert, Methods of mathematical physics. Vol. I. Interscience Publishers, Inc., New York, N.Y., 1953.

[15] H. Donnelly and C. Fefferman, "Nodal sets of eigenfunctions: Riemannian manifolds with boundary," in Analysis, et cetera, pp. 251-262, Boston, MA: Academic Press, 1990.

[16] H. Donnelly and C. Fefferman, "Growth and geometry of eigenfunctions of the Laplacian," in Analysis and partial differential equations, vol. 122 of Lecture Notes in Pure and Appl. Math., pp. 635-655, New York: Dekker, 1990.

[17] H. Donnelly and C. Fefferman, "Nodal sets for eigenfunctions of the Laplacian on surfaces," J. Amer. Math. Soc., vol. 3, no. 2, pp. 333-353, 1990.

[18] H. Donnelly and C. Fefferman, "Nodal domains and growth of harmonic functions on noncompact manifolds," J. Geom. Anal., vol. 2, no. 1, pp. 79-93, 1992.

[19] H. Donnelly and C. Fefferman, "Nodal sets of eigenfunctions on Riemannian manifolds," Invent. Math., vol. 93, no. 1, pp. 161-183, 1988.

[20] L. Friedlander, "On the second eigenvalue of the Dirichlet Laplacian," Israel J. Math., vol. 79, no. 1, pp. 23-32, 1992.

[21] D. Fujiwara, "A remark on the Hadamard variational formula. II," Proc. Japan Acad. Ser. A Math. Sci., vol. 57, no. 7, pp. 337-341, 1981.

[22] D. Fujiwara and S. Ozawa, "The Hadamard variational formula for the Green functions of some normal elliptic boundary value problems," Proc. Japan Acad. Ser. A Math. Sci., vol. 54, no. 8, pp. 215-220, 1978.

[23] Galileo Galilei, Dialogues Concerning Two New Sciences (English translation), Chicago: The MacMillan Company 1939.

[24] P. R. Garabedian, Partial differential equations. New York: Chelsea Publishing Co., second ed., 1986.

[25] P. R. Garabedian and M. Schiffer, "Convexity of domain functionals," J. Analyse Math., vol. 2, pp. 281-368, 1953.

[26] N. Ghoussoub, "Location, multiplicity and Morse indices of min-max critical points," J. Reine Angew. Math., vol. 417, pp. 27-76, 1991.

[27] S. Gnutzmann, U. Smilansky, and N. Sondergaard. Resolving isospectral 'drums' by counting nodal domains. J. Phys. A, 38(41):8921-8933, 2005.

[28] P. Grinfeld, "Hadamards formula inside and out," J. Optim. Theory Appl., vol. 146, 2010.

[29] J. Hadamard, Mémoire sur le problème danalyse relatif à léquilibre des plaques elastiques encastrées. Mem. Acad. Sci. Inst. de France, 1908.

[30] O. H. Hald and J. R. McLaughlin, Inverse Nodal Problems: Finding the Potential From Nodal Lines, Mem. AMS, v. 119, no. 572, 1996.

[31] B. Helffer, T. Hoffmann-Ostenhof, and S. Terracini, "Nodal domains and spectral minimal partitions," Ann. Inst. H. Poincaré Anal. Non Linéaire, vol. 26, no. 1, pp. 101-138, 2009. 
[32] B. Helffer, "Domaines nodaux et partitions spectrales minimales (d'après B. Helffer, T. Hoffmann-Ostenhof et S. Terracini)," in Séminaire: Équations aux Dérivées Partielles. 2006-200\%, Sémin. Équ. Dériv. Partielles, pp. Exp. No. VIII, 23, Palaiseau: École Polytech., 2007.

[33] H. Hezari, S. Zelditch, $C^{\infty}$ spectral rigidity of the ellipse, preprint arXiv:1007.1741,

[34] D. Hilbert, Grundzüge einer allgemainen Theorie der linearen Integralgleichungen. Leipzig, 1912.

[35] D. Husemoller, Fibre Bundles, McGraw - Hill 1966.

[36] L. Ivanov, L. Kotko, and S. Kreı̆n, "Boundary value problems in variable domains," Differencial'nye Uravnenija i ikh Primenenie, Vilnus, no. 19, pp. 1-161, 1977.

[37] P. D. Karageorge and U. Smilansky. Counting nodal domains on surfaces of revolution. J. Phys. A, 41(20):205102, 26, 2008.

[38] D. Klawonn. Inverse nodal problems. J. Phys. A, 42(17):175209, 11, 2009.

[39] V. Kozlov, "On the Hadamard formula for nonsmooth domains," J. Differential Equations, vol. 230 (2006), no. 2, pp. 532-555,

[40] S. Lang, Introduction to differentiable manifolds. Universitext, New York: SpringerVerlag, second ed., 2002.

[41] J.-L. Lions and E. Magenes, Non-homogeneous boundary value problems and applications. Vol. I. New York: Springer-Verlag, 1972. Translated from the French by P. Kenneth, Die Grundlehren der mathematischen Wissenschaften, Band 181.

[42] E. MacCurdy Notebooks of Leonardo da Vinci, Jonathtan Cape, London 1938.

[43] J. R. McLaughlin, Good Vibrations, American Scientist 86 (1998), no. 4, 342-349.

[44] A. M. Micheletti, "Perturbazione dello spettro di un operatore ellittico di tipo variazionale, in relazione ad una variazione del campo," Ann. Mat. Pura Appl. (4), vol. 97, pp. 267-281, 1973.

[45] A. M. Micheletti, "Perturbazione dello spettro dell'operatore di Laplace, in relazione ad una variazione del campo," Ann. Scuola Norm. Sup. Pisa (3), vol. 26, pp. 151-169, 1972.

[46] N. Nadirashvili, D. Tot, and D. Yakobson, "Geometric properties of eigenfunctions," Uspekhi Mat. Nauk, vol. 56, no. 6(342), pp. 67-88, 2001.

[47] Oxford Dictionary of Scientists, Oxford Univ. Press 1999, p. 101.

[48] J. Peetre, "On Hadamard's variational formula," J. Differential Equations, vol. 36, no. 3, pp. 335-346, 1980.

[49] Å. Pleijel, "Remarks on Courant's nodal line theorem," Comm. Pure Appl. Math., vol. 9, pp. 543-550, 1956.

[50] J. Rayleigh, The Theory of Sound. Dover Publications, New York, N. Y., 1945. 2d ed.

[51] K. Uhlenbeck, "Generic properties of eigenfunctions," Amer. J. Math., vol. 98, no. 4, pp. 1059-1078, 1976.

[52] M. G. Zaŭdenberg, S. G. Krĕnn, P. A. Kučment, and A. A. Pankov, "Banach bundles and linear operators," Uspehi Mat. Nauk, vol. 30, no. 5(185), pp. 101-157. English translation: Russian Math. Surveys 30 (1975), no. 5, 115-175, 1975.

${ }^{1}$ Department of Mathematics, Texas A\&M University, College Station, TX 77843-3368 USA.

2 Department of Physics of Complex Systems, Weizmann Institute of ScIENCE, Rehovot 76100, IsRael.

${ }^{3}$ School of Mathematics, Cardiff University, Cardiff, Wales, UK 